Article

\title{
Essential Oil Compositions of Three Invasive Conyza Species Collected in Vietnam and Their Larvicidal Activities against Aedes aegypti, Aedes albopictus, and Culex quinquefasciatus
}

\author{
Tran Minh Hoi ${ }^{1}$, Le Thi Huong $\left.{ }^{2}{ }^{(}\right)$, Hoang Van Chinh ${ }^{3}$, Dang Viet Hau ${ }^{4}$, Prabodh Satyal ${ }^{5}$, \\ Thieu Anh Tai ${ }^{6}$, Do Ngoc Dai ${ }^{7,8}{ }^{\mathbb{C}}$, Nguyen Huy Hung ${ }^{6,9, *} \mathbb{C}, \mathrm{Vu}$ Thi Hien ${ }^{10}$ and \\ William N Setzer $5,11, *$ (D) \\ 1 Department of Plant Resources, Institute of Ecology and Biological Resources, Vietnam Academy of Science \\ and Technology, Hanoi 100000, Vietnam; tranhoiiebr@gmail.com \\ 2 School of Natural Science Education, Vinh University, 182 Le Duan, Vinh City 43000, Vietnam; \\ lehuong223@gmail.com \\ 3 Faculty of Natural Sciences, Hong Duc University, 365 Quang Trung, Thanh Hoa 440000, Vietnam; \\ hoangvanchinh@hdu.edu.vn \\ 4 Center for Research and Technology Transfer, Vietnam Academy of Science and Technology, \\ Hanoi 100000, Vietnam; hauhoahock20@gmail.com \\ 5 Aromatic Plant Research Center, 230 N 1200 E, Suite 102, Lehi, UT 84043, USA; psatyal@aromaticplant.org \\ 6 Department of Pharmacy, Duy Tan University, 03 Quang Trung, Da Nang 550000, Vietnam; \\ anhtai0808qn@gmail.com \\ 7 Graduate University of Science and Technology, Vietnam Academy of Science and Technology, \\ 18-Hoang Quoc Viet, Cau Giay, Hanoi, 100000 Vietnam; daidn23@gmail.com \\ 8 Faculty of Agriculture, Forestry and Fishery, Nghe An College of Economics, 51-Ly Tu Trong, \\ Vinh City 460000, Vietnam \\ 9 Center for Advanced Chemistry, Institute of Research and Development, Duy Tan University, \\ 03 Quang Trung, Da Nang 550000, Vietnam \\ 10 Faculty of Hydrometerology, Ho Chi Minh City University of Natural Resources and Environment, \\ Ho Chi Minh City 70000, Vietnam; hiensphoa@gmail.com \\ 11 Department of Chemistry, University of Alabama in Huntsville, Huntsville, AL 35899, USA \\ * Correspondence: nguyenhuyhung@duytan.edu.vn (N.H.H.); wsetzer@chemistry.uah.edu (W.N.S.)
}

Academic Editor: Giovanni Benelli

Received: 20 September 2020; Accepted: 6 October 2020; Published: 7 October 2020

\begin{abstract}
Mosquito-borne infectious diseases are a persistent problem in tropical regions of the world, including Southeast Asia. Vector control has relied principally on synthetic insecticides, but these have detrimental environmental effects and there is an increasing demand for plant-based agents to control insect pests. Invasive weedy plant species may be able to serve as readily available sources of essential oils, some of which may be useful as larvicidal agents for control of mosquito populations. We hypothesize that members of the genus Conyza (Asteraceae) may produce essential oils that may have mosquito larvicidal properties. The essential oils from the aerial parts of Conyza bonariensis, C. canadensis, and C. sumatrensis were obtained by hydrodistillation, analyzed by gas chromatography-mass spectrometry, and screened for mosquito larvicidal activity against Aedes aegypti, Ae. albopictus and Culex quinquefasciatus. The essential oils of $C$. canadensis and C. sumatrensis, both rich in limonene ( $41.5 \%$ and $25.5 \%$, respectively), showed notable larvicidal activities against $A$ e. aegypti (24-h $\mathrm{LC}_{50}=9.80$ and $21.7 \mu \mathrm{g} / \mathrm{mL}$, respectively) and Ae. albopictus (24-h $\mathrm{LC}_{50}=18.0$ and $19.1 \mu \mathrm{g} / \mathrm{mL}$, respectively). These two Conyza species may, therefore, serve as sources for alternative, environmentally-benign larvicidal control agents.
\end{abstract}


Keywords: Erigeron; Conyza bonariensis; Conyza canadensis; Conyza sumatrensis; mosquito; vector control

\section{Introduction}

Mosquito-borne infectious diseases have been a continuous health problem in Southeast Asia, including Vietnam. Dengue fever and dengue hemorrhagic fever are particularly problematic and chikungunya fever is an emerging threat in the country [1,2]. Aedes aegypti (L.) (Diptera: Culicidae), the yellow fever mosquito, is a recognized vector of dengue fever virus, chikungunya fever virus, Zika virus, and yellow fever virus [3]. Aedes albopictus (Skuse) (Diptera: Culicidae), the Asian tiger mosquito, is a key vector of several pathogenic viruses, including yellow fever virus [4], dengue fever virus [5], chikungunya virus [6], and possibly Zika virus [7]. Culex quinquefasciatus Say (Diptera: Culicidae), the southern house mosquito, is a vector of lymphatic filariasis [8] as well as several arboviruses such as West Nile virus and St. Louis encephalitis virus [9] and possibly Zika virus [10].

Several members of the genus Conyza Less. (Asteraceae) have been introduced throughout the tropics and subtropics where they have become invasive weeds [11-13]. Conyza bonariensis (L.) Cronquist (syn. Erigeron bonariensis L.), flaxleaf fleabane, probably originated in South America [14], but has been introduced throughout Asia, Africa, Mexico and the southern United States, Europe, and Oceania $[13,15]$. Conyza canadensis (L.) Cronquist (syn. Erigeron canadensis L.), Canada fleabane, is native to North America, but is also now naturalized throughout Europe, Asia, and Oceania [13]. Conyza sumatrensis (Retz.) E. Walker (syn. Erigeron sumatrensis Retz.) is probably native to South America, but this species has also been naturalized in tropical and subtropical regions [16].

Non-native invasive plant species are generally detrimental to the local environments where they have been introduced. They can outcompete native plant species and reduce biodiversity [17], they can alter ecosystem functions [18], and can have substantial economic impacts [19]. Control methods for invasive plants have generally included application of herbicides, physical cutting, or burning [20]. However, harvesting invasive species for beneficial uses as a method for control of invasive species may provide economic incentives to offset eradication costs [21]. For example, Melaleuca quinquinervia trees in south Florida have been cut and chipped for landscape mulch and boiler fuel [22]; it has been suggested that mechanical harvesting of invasive cattail (Typha spp.), common reed (Phragmites australis), and reed canary grass (Phalaris arundinacea) from coastal wetlands of Lake Ontario can be used as an agricultural nutrient source or as a biofuel [23]. The leaf essential oil of Solidago canadensis, an invasive plant in Europe, has been evaluated as a potential insecticide and demonstrated moderate larvicidal activity against $C x$. quinquefasciatus [24].

The use of synthetic pesticides for mosquito control has had detrimental effects on the environment $[25,26]$. They tend to be persistent, toxic to non-target organisms, and insecticide resistance has been steadily increasing in mosquito species [27]. Essential oils have been suggested as viable, environmentally benign, and renewable alternatives to synthetic pesticides [28-32]. We have recently studied several introduced invasive plant species in Vietnam for potential use as mosquito vector control agents [33-35], and as part of our ongoing efforts in identifying readily-available essential oils for mosquito control, we have examined three Conyza species for larvicidal activity against Aedes aegypti, Aedes albopictus, and Culex quinquefasciatus, with the aim of identifying new mosquito-control essential oils and the components responsible for the activity.

\section{Results and Discussion}

\subsection{Essential Oil Compositions}

The essential oils from the aerial parts of $C$. bonariensis, $C$. canadensis, and C. sumatrensis were obtained by hydrodistillation in $1.10 \%, 1.37 \%$, and $1.21 \%$ yield. The chemical compositions of the Conyza essential oils, determined using gas chromatography-mass spectrometry, are summarized in Table 1. 
Conyza bonariensis essential oil was dominated by sesquiterpenoids, especially allo-aromadendrene $(41.2 \%), \beta$-caryophyllene (13.3\%), and caryophyllene oxide (12.2\%). Concentrations of monoterpenoids $(1.8 \%)$ and diterpenoids (trace) were relatively small. The essential oils of $C$. canadensis and C. sumatrensis, on the other hand, were rich in limonene ( $41.5 \%$ and $25.5 \%$, respectively). The aerial parts essential oil of C. sumatrensis also had a large concentration of (Z)-lachnophyllum ester (20.7\%). There is wide variation in the essential oil compositions of Conyza species, both between species and within the same species (see Table 2). This is not surprising given the very different geographical locations of the collection sites for these samples.

Table 1. Chemical compositions of the aerial parts essential oils of Conyza bonariensis, Conyza canadensis, and Conyza sumatrensis collected in Vietnam.

\begin{tabular}{|c|c|c|c|c|c|}
\hline \multirow{2}{*}{$\mathrm{RI}_{\text {calc }}{ }^{\mathrm{a}}$} & \multirow{2}{*}{$R I_{d b} b$} & \multirow{2}{*}{ Compound } & \multicolumn{3}{|c|}{ Relative Content $\%$} \\
\hline & & & C. bonariensis & C. canadensis & C. sumatrensis \\
\hline 931 & 932 & $\alpha$-Pinene & 0.5 & 0.5 & 0.2 \\
\hline 948 & 950 & Camphene & $\operatorname{tr}^{\mathrm{c}}$ & - & - \\
\hline 967 & 972 & (3Z)-Octen-2-ol & - & - & $\operatorname{tr}$ \\
\hline 971 & 972 & Sabinene & $\operatorname{tr}$ & 0.1 & 0.1 \\
\hline 976 & 978 & $\beta$-Pinene & 0.8 & 8.8 & 3.0 \\
\hline 982 & 984 & 6-Methylhept-5-en-2-one & - & - & $\operatorname{tr}$ \\
\hline 987 & 989 & Myrcene & $\operatorname{tr}$ & 1.2 & 1.0 \\
\hline 1023 & 1025 & p-Cymene & $\operatorname{tr}$ & 0.3 & 0.1 \\
\hline 1028 & 1030 & Limonene & 0.2 & 41.5 & 25.5 \\
\hline 1030 & 1031 & $\beta$-Phellandrene & - & $\operatorname{tr}$ & - \\
\hline 1034 & 1034 & (Z)- $\beta$-Ocimene & - & - & $\operatorname{tr}$ \\
\hline 1044 & 1045 & $(E)$ - $\beta$-Ocimene & - & $\operatorname{tr}$ & 1.9 \\
\hline 1049 & 1051 & 2,3,6-Trimethylhepta-1,5-diene & - & $\operatorname{tr}$ & - \\
\hline 1056 & 1057 & $\gamma$-Terpinene & - & $\operatorname{tr}$ & - \\
\hline 1088 & 1091 & p-Cymenene & - & 0.1 & - \\
\hline 1090 & 1091 & Rosefuran & - & - & 0.1 \\
\hline 1093 & 1097 & $\alpha$-Pinene oxide & - & - & 0.2 \\
\hline 1097 & 1098 & Perillene & - & 0.1 & - \\
\hline 1098 & 1101 & Linalool & 0.2 & - & - \\
\hline 1101 & 1101 & 6-Methyl-3,5-heptadien-2-one & - & - & 0.1 \\
\hline 1103 & 1104 & Nonanal & $\operatorname{tr}$ & - & - \\
\hline 1112 & 1113 & 4,8-Dimethylnona-1,3,7-triene & - & - & 0.2 \\
\hline 1118 & 1119 & endo-Fenchol & $\operatorname{tr}$ & - & - \\
\hline 1120 & 1121 & trans- $p$-Mentha-2,8-dien-1-ol & - & 0.9 & 0.2 \\
\hline 1124 & 1131 & Cyclooctanone & - & 0.8 & - \\
\hline 1129 & 1130 & 4-Acetyl-1-methylcyclohexene & - & 0.1 & - \\
\hline 1131 & 1132 & cis-Limonene oxide & - & 0.6 & 0.2 \\
\hline 1134 & 1137 & cis-p-Mentha-2,8-dien-1-ol & - & 1.2 & 0.3 \\
\hline 1135 & 1137 & trans-Limonene oxide & - & 0.6 & - \\
\hline 1137 & 1137 & Nopinone & - & 0.4 & - \\
\hline 1137 & 1139 & (E)-Myroxide & - & - & 0.1 \\
\hline 1139 & 1141 & trans-Pinocarveol & $\operatorname{tr}$ & 1.6 & 0.1 \\
\hline 1150 & 1152 & Citronellal & - & 0.1 & - \\
\hline 1160 & 1164 & Pinocarvone & - & 0.8 & $\operatorname{tr}$ \\
\hline 1170 & 1170 & Borneol & $\operatorname{tr}$ & - & - \\
\hline 1177 & 1179 & 2-Isopropenyl-5-methylhex-4-enal & - & 0.3 & - \\
\hline 1182 & 1184 & $p$-Methylacetophenone & - & 0.3 & - \\
\hline 1185 & 1185 & Cryptone & - & 0.4 & - \\
\hline 1185 & 1187 & trans- $p$-Mentha-1(7),8-dien-2-ol & - & 0.2 & - \\
\hline 1189 & 1190 & Methyl salicylate & $\operatorname{tr}$ & - & - \\
\hline 1193 & 1195 & $\alpha$-Terpineol & 0.1 & - & 0.1 \\
\hline 1193 & 1196 & Myrtenal & - & 1.4 & - \\
\hline 1194 & 1195 & Myrtenol & - & 1.2 & - \\
\hline 1196 & 1197 & Methyl chavicol (=Estragol) & - & 0.2 & - \\
\hline 1198 & 1201 & cis-Piperitol & - & 0.8 & 0.1 \\
\hline 1206 & 1207 & Oct-3E-enyl acetate & - & - & 0.1 \\
\hline 1217 & 1218 & trans-Carveol & - & 3.8 & 0.2 \\
\hline
\end{tabular}


Table 1. Cont.

\begin{tabular}{|c|c|c|c|c|c|}
\hline \multirow{2}{*}{$\mathrm{RI}_{\text {calc }}{ }^{\mathrm{a}}$} & \multirow{2}{*}{$R I_{d b} b$} & \multirow{2}{*}{ Compound } & \multicolumn{3}{|c|}{ Relative Content $\%$} \\
\hline & & & C. bonariensis & C. canadensis & C. sumatrensis \\
\hline 1227 & 1228 & cis-p-Mentha-1(7),8-dien-2-ol & - & 0.1 & - \\
\hline 1230 & 1232 & cis-Carveol & - & 1.1 & 0.1 \\
\hline 1242 & 1242 & Carvone & - & 3.8 & 0.2 \\
\hline 1247 & 1249 & Linalyl acetate & $\operatorname{tr}$ & - & - \\
\hline 1266 & 1270 & iso-Piperitenone & - & 0.6 & - \\
\hline 1273 & 1277 & Perilla aldehyde & - & 0.5 & - \\
\hline 1287 & 1287 & Limonene dioxide & - & 0.7 & - \\
\hline 1296 & 1299 & Perilla alcohol & - & 0.4 & - \\
\hline 1303 & - & Unidentified $^{\mathrm{d}}$ & - & 1.1 & - \\
\hline 1316 & 1324 & Limonene hydroperoxide & - & 1.1 & - \\
\hline 1343 & 1346 & Limonene-1,2-diol & - & 2.6 & - \\
\hline 1344 & 1349 & 7-epi-Silphiperfol-5-ene & - & - & 0.3 \\
\hline 1345 & 1349 & $\alpha$-Cubebene & 0.2 & - & - \\
\hline 1355 & 1340 & $p$-Mentha-6,8-diene-2-hydroperoxide & - & 1.2 & - \\
\hline 1367 & 1371 & $\alpha$-Ylangene & $\operatorname{tr}$ & - & - \\
\hline 1374 & 1375 & $\alpha$-Copaene & 4.5 & - & 0.1 \\
\hline 1376 & 1380 & Daucene & - & - & 0.4 \\
\hline 1377 & 1374 & Isoledene & - & - & 0.3 \\
\hline 1379 & 1382 & Modheph-2-ene & - & - & 0.4 \\
\hline 1381 & 1382 & $\beta$-Bourbonene & $\operatorname{tr}$ & - & - \\
\hline 1385 & 1387 & $\beta$-Cubebene & 0.4 & - & 0.1 \\
\hline 1386 & 1385 & $\alpha$-Isocomene & - & - & 0.1 \\
\hline 1387 & 1390 & $\beta$-Elemene & 0.3 & - & 0.4 \\
\hline 1392 & 1394 & Sativene & - & - & 0.1 \\
\hline 1398 & 1405 & (Z)-Caryophyllene & 0.2 & - & - \\
\hline 1404 & 1406 & $\alpha$-Gurjunene & 0.1 & - & - \\
\hline 1408 & 1411 & $\beta$-Isocomene & - & - & 0.1 \\
\hline 1418 & 1417 & (E)-Caryophyllene & 13.3 & - & 5.5 \\
\hline 1427 & 1430 & $\beta$-Copaene & 0.2 & - & 0.2 \\
\hline 1430 & 1433 & trans- $\alpha$-Bergamotene & - & - & 1.1 \\
\hline 1432 & 1440 & 6,9-Guaiadiene & - & - & 0.2 \\
\hline 1433 & 1436 & $\alpha$-Guaiene & 1.8 & - & - \\
\hline 1436 & 1438 & Aromadendrene & 0.2 & - & 0.1 \\
\hline 1445 & 1449 & (E)-Lachnophyllum acid & - & - & 0.2 \\
\hline 1451 & 1452 & (E)- $\beta$-Farnesene & - & - & 6.7 \\
\hline 1453 & 1454 & $\alpha$-Humulene & 5.4 & 0.3 & 0.7 \\
\hline 1457 & 1463 & cis-Cadina-1(6),4-diene & - & - & 0.4 \\
\hline 1460 & 1458 & allo-Aromadendrene & 41.2 & - & - \\
\hline 1469 & - & Unidentified $^{\mathrm{e}}$ & - & - & 1.3 \\
\hline 1472 & 1472 & trans-Cadina-1(6),4-diene & 0.5 & - & 0.2 \\
\hline 1476 & 1479 & $\alpha$-Amorphene & 0.1 & - & - \\
\hline 1478 & 1483 & Germacrene D & 0.3 & - & 2.1 \\
\hline 1481 & 1483 & trans- $\beta$-Bergamotene & - & - & 0.2 \\
\hline 1486 & 1489 & $\beta$-Selinene & 0.5 & - & - \\
\hline 1488 & 1491 & Viridiflorene & 0.2 & - & - \\
\hline 1492 & 1497 & Bicyclogermacrene & - & - & 0.3 \\
\hline 1493 & 1497 & $\alpha$-Selinene & 0.3 & - & - \\
\hline 1495 & 1497 & $\alpha$-Muurolene & 0.4 & - & 0.1 \\
\hline 1498 & 1505 & $\alpha$-Bulnesene & 1.8 & - & - \\
\hline 1501 & 1505 & $(E, E)-\alpha$-Farnesene & - & - & 0.1 \\
\hline 1504 & 1514 & (Z)-Lachnophyllum acid & - & 0.2 & 0.8 \\
\hline 1507 & 1510 & (E)-Lachnophyllum ester & - & - & 0.4 \\
\hline 1510 & 1512 & $\gamma$-Cadinene & 0.4 & - & 0.1 \\
\hline 1515 & 1515 & (Z)-Lachnophyllum ester & - & 5.5 & 20.7 \\
\hline 1515 & 1518 & $\delta$-Cadinene & 0.6 & - & - \\
\hline 1518 & 1519 & trans-Calamenene & 0.3 & - & - \\
\hline 1521 & 1523 & $\beta$-Sesquiphellandrene & - & - & 0.3 \\
\hline 1531 & 1532 & Tridec-11-yn-1-ol & - & - & 0.3 \\
\hline 1533 & 1538 & $\alpha$-Cadinene & 0.1 & - & - \\
\hline 1538 & 1541 & $\alpha$-Calacorene & 0.1 & - & - \\
\hline
\end{tabular}


Table 1. Cont.

\begin{tabular}{|c|c|c|c|c|c|}
\hline \multirow{2}{*}{$\mathrm{RI}_{\text {calc }}{ }^{\mathrm{a}}$} & \multirow{2}{*}{$\mathrm{RI}_{\mathrm{db}} \mathrm{b}$} & \multirow{2}{*}{ Compound } & \multicolumn{3}{|c|}{ Relative Content $\%$} \\
\hline & & & C. bonariensis & C. canadensis & C. sumatrensis \\
\hline 1556 & 1557 & Germacrene B & - & - & 0.1 \\
\hline 1558 & 1560 & (E)-Nerolidol & - & 0.2 & 1.8 \\
\hline 1559 & 1564 & $\beta$-Calacorene & 0.1 & - & - \\
\hline 1565 & 1566 & 1,5-Epoxysalvial-4(14)-ene & - & - & 0.2 \\
\hline 1566 & 1568 & Dendrolasin & - & - & 0.1 \\
\hline 1567 & 1567 & Palustrol & 0.1 & - & - \\
\hline 1574 & 1576 & Spathulenol & 1.3 & - & 5.2 \\
\hline 1580 & 1577 & Caryophyllene oxide & 12.2 & 1.1 & 5.8 \\
\hline 1582 & 1590 & Globulol & 0.4 & - & 0.5 \\
\hline 1589 & 1593 & Salvial-4(14)-en-1-one & - & 0.1 & 0.2 \\
\hline 1590 & 1594 & Viridiflorol & 0.8 & - & 0.3 \\
\hline 1593 & 1599 & Cubeban-11-ol & 0.2 & - & - \\
\hline 1599 & 1601 & Carotol & - & - & 1.1 \\
\hline 1601 & 1605 & Ledol & 0.6 & - & - \\
\hline 1606 & 1611 & Humulene epoxide II & 2.2 & 2.9 & 0.4 \\
\hline 1624 & 1628 & 1-epi-Cubenol & 0.2 & - & - \\
\hline 1629 & 1629 & iso-Spathulenol & - & - & 0.6 \\
\hline 1633 & 1635 & Caryophylla-4(12),8(13)-dien-5 $\beta$-ol & 0.2 & - & - \\
\hline 1635 & 1632 & Muurola-4,10(14)-dien-1 $\beta$-ol & - & - & 0.7 \\
\hline 1638 & 1643 & $\tau$-Cadinol & 0.2 & - & 0.4 \\
\hline 1640 & 1644 & $\tau$-Muurolol & 0.1 & - & 0.3 \\
\hline 1643 & 1643 & $\alpha$-Muurolol & 0.2 & - & - \\
\hline 1643 & 1644 & allo-Aromadendrene epoxide & - & 0.3 & - \\
\hline 1652 & 1655 & $\alpha$-Cadinol & 0.6 & 0.3 & 0.4 \\
\hline 1655 & 1655 & Eudesma-4(15),7-dien-1 $\alpha$-ol & - & - & 0.1 \\
\hline 1661 & 1664 & cis-Calamenen-10-ol & 0.1 & - & - \\
\hline 1666 & 1666 & 14-Hydroxy-9-epi-(E)-caryophyllene & 0.1 & - & - \\
\hline 1669 & 1677 & Cadalene & 0.1 & - & - \\
\hline 1686 & 1685 & Eudesma-4(15),7-dien-1 $\beta$-ol & - & 0.4 & 0.1 \\
\hline 1698 & 1704 & cis-Thujopsenol & 0.1 & - & - \\
\hline 1717 & - & Unidentified $^{f}$ & - & 1.0 & - \\
\hline 1738 & 1740 & $8 \alpha, 11$-Elemodiol & 0.1 & - & - \\
\hline 1751 & 1748 & Khusimol & 1.5 & - & - \\
\hline 1790 & 1792 & 14 -Hydroxy- $\delta$-cadinene & - & - & 0.2 \\
\hline 1800 & - & Unidentified $\mathrm{g}$ & 1.1 & - & - \\
\hline 1833 & 1836 & Neophytadiene & - & - & 0.2 \\
\hline 1857 & 1860 & Platambin & 0.1 & 0.5 & 0.1 \\
\hline 1882 & 1884 & Corymbolone & 0.2 & - & - \\
\hline \multirow[t]{8}{*}{2103} & 2102 & Phytol & $\operatorname{tr}$ & - & 0.1 \\
\hline & & Monoterpene hydrocarbons & 1.5 & 52.7 & 31.8 \\
\hline & & Oxygenated monoterpenoids & 0.3 & 26.4 & 1.9 \\
\hline & & Sesquiterpene hydrocarbons & 73.7 & 0.3 & 20.7 \\
\hline & & Oxygenated sesquiterpenoids & 21.3 & 5.7 & 18.5 \\
\hline & & Diterpenoids & trace & - & 0.4 \\
\hline & & Others & trace & 7.2 & 22.9 \\
\hline & & Total Identified & 96.8 & 92.3 & 96.1 \\
\hline
\end{tabular}

${ }^{\mathrm{a}} \mathrm{RI}_{\text {calc }}=$ Retention Index calculated with respect to a homologous series of n-alkanes on a ZB- 5 column. ${ }^{\mathrm{b}} \mathrm{RI}_{\mathrm{db}}=$ Retention Index from the databases [36-39]. ${ }^{c} \mathrm{tr}=\operatorname{trace}(<0.05 \%) .{ }^{\mathrm{d}} \mathrm{MS}(\mathrm{EI}): 150(3 \%), 135(51 \%), 121(29 \%), 119(38 \%)$, 109(42\%), 107(66\%), 93(97\%), 91(89\%), 81(50\%), 79(100\%), 69(82\%), 67(37\%), 55(65\%), 53(40\%), 43(75\%), 41(85\%). e MS(EI): 204(25\%), 189(3\%), 161(100\%), 147(9\%), 133(28\%), 120(48\%), 119(25\%), 105(51\%), 91(47\%), 69(20\%), 57(19\%), 55(21\%), 41(20\%). ${ }^{\mathrm{f}} \mathrm{MS}(\mathrm{EI}): 175(3 \%), 135(11 \%), 111(48 \%), 93(20 \%), 83(19 \%), 67(19 \%), 55(26 \%), 43(100 \%), 41(20 \%)$ g MS(EI): 218(29\%), 203(28\%), 189(100\%), 175(46\%), 147(34\%), 133(61\%), 119(38\%), 105(70\%), 91(90\%), 79(42\%), $67(43 \%), 55(34 \%), 41(52 \%)$. 
Table 2. Major components of Conyza bonariensis, Conyza canadensis, and Conyza sumatrensis essential oils from different geographical locations.

\begin{tabular}{|c|c|c|}
\hline Conyza Species (Collection Site) & Major Components (>5\%) & Ref. \\
\hline C. bonariensis aerial parts EO (Chapada dos Guimarães, Mato Grosso, Brazil) & $\begin{array}{l}\text { limonene }(6.9 \%),(E) \text {-caryophyllene }(14.4 \%),(E) \text { - } \beta \text {-farnesene }(23.3 \%) \text {, } \\
\text { germacrene D }(15.3 \%) \text {, bicyclogermacrene }(8.3 \%) \text {, spathulenol }(7.6 \%)\end{array}$ & [40] \\
\hline C. bonariensis aerial parts EO (Melgaço, Pará, Brazil) & $\begin{array}{l}\text { limonene }(22.9 \%),(E) \text {-caryophyllene }(13.3 \%) \text {, trans- } \alpha \text {-bergamotene }(5.3 \%) \text {, } \\
(E) \text { - } \beta \text {-farnesene }(20.1 \%) \text {, bicyclogermacrene }(6.6 \%) \text {, spathulenol }(6.3 \%)\end{array}$ & {$[40]$} \\
\hline C. bonariensis aerial parts EO (Peixe-Boi, Pará, Brazil) & $(E)$-caryophyllene $(13.3 \%)$, trans- $\alpha$-bergamotene $(8.1 \%),(E)$ - $\beta$-farnesene $(30.9 \%)$ & [40] \\
\hline C. bonariensis aerial parts EO (alta Floresta, Mato Grosso, Brazil) & $\begin{array}{l}\text { limonene }(12.6 \%),(E) \text {-caryophyllene }(13.0 \%),(E)-\beta \text {-farnesene }(19.1 \%) \text {, } \\
\text { germacrene } \mathrm{D}(13.2 \%) \text {, bicyclogermacrene }(6.3 \%) \text {, spathulenol }(5.7 \%)\end{array}$ & {$[40]$} \\
\hline C. bonariensis aerial parts EO (Macapá, Amapá, Brazil) & limonene (58.4\%), (E)- $\beta$-farnesene (7.0\%) & [40] \\
\hline C. bonariensis aerial parts EO (Rio de Janeiro, Brazil) & limonene $(45.0 \%),(E)-\beta$-ocimene $(13.0 \%),(E)-\beta$-farnesene $(6.6 \%)$, germacrene $\mathrm{D}(6.4 \%)$ & {$[41]$} \\
\hline C. bonariensis leaf EO (Minas Gerais State, Brazil) & $\begin{array}{l}\text { limonene }(29.6 \%) \text {, trans- } \alpha \text {-bergamotene }(10.3 \%) \text {, matricaria methyl ester }(8.3 \%) \text {, } \\
\beta \text {-copaen- } 4 \alpha-\text { ol }(7.4 \%)\end{array}$ & [42] \\
\hline C. bonariensis aerial parts EO (Athens, Greece) & $\begin{array}{l}\text { limonene }(8.3 \%),(E) \text { - } \beta \text {-ocimene }(11.5 \%),(E) \text { - } \beta \text {-farnesene }(8.1 \%),(Z) \text {-lachnophyllum } \\
\text { ester }(21.2 \%) \text {, matricaria ester }(17.5 \%)\end{array}$ & [43] \\
\hline C. bonariensis aerial parts EO (Southwestern Misiones Province, Argentina) & $\begin{array}{l}\text { limonene }(13.5 \%),(E) \text { - } \beta \text {-ocimene }(13.3 \%), p \text {-mentha-1,3,8-triene }(5.2 \%) \text {, } \\
\text { germacrene } \mathrm{D}(14.6 \%) \text {, bicyclogermacrene }(6.6 \%)\end{array}$ & {$[44]$} \\
\hline C. bonariensis leaf EO (Monastir, Tunisia) & $\begin{array}{l}\text { limonene }(5.8 \%) \text {, terpinolene }(5.3 \%),(E) \text { - } \beta \text {-farnesene }(7.5 \%) \text {, matricaria ester }(17.8 \%) \text {, } \\
\text { caryophyllene oxide }(7.8 \%)\end{array}$ & [45] \\
\hline C. bonariensis aerial parts EO (Cagliari, Sardinia, Italy) & $\begin{array}{l}\text { limonene }(5.1 \%) \text {, carvacrol }(9.8 \%), \alpha \text {-curcumene }(10.2 \%) \text {, spathulenol }(18.6 \%) \text {, } \\
\text { caryophyllene oxide }(18.7 \%) \text {, neophytadiene }(6.1 \%)\end{array}$ & [46] \\
\hline C. bonariensis leaf EO (Mérida State, Venezuela) & $\begin{array}{l}\text { limonene }(5.1 \%),(Z)-\beta \text {-ocimene }(5.1 \%),(E) \text { - } \beta \text {-ocimene }(20.7 \%) \\
(E) \text { - } \beta \text {-farnesene }(37.8 \%), \alpha \text {-farnesene }(5.6 \%), \beta \text {-sesquiphellandrene }(9.8 \%)\end{array}$ & [47] \\
\hline C. bonariensis leaf EO (Kabianga, Kericho, Kenya) & $\begin{array}{l}\text { ß-pinene }(5.4 \%) \text {, limonene }(8.3 \%) \text {, } \\
\text { 2,6,7,7a-tetrahydro-1,5-dimethyl-1H-indene-3-carboxaldehyde }(49.1 \%)^{a}\end{array}$ & {$[48]$} \\
\hline C. bonariensis aerial parts EO (Parana State, Brazil) & limonene (66.3\%), 2-heptyl acetate $(6.9 \%)$ & [49] \\
\hline C. bonariensis aerial parts EO & $\begin{array}{l}\text { (E)-caryophyllene }(13.3 \%), \alpha \text {-humulene }(5.4 \%) \text {, allo-aromadendrene }(41.2 \%) \text {, } \\
\text { caryophyllene oxide }(12.2 \%)\end{array}$ & this work \\
\hline C. canadensis aerial parts EO (Plovdiv, Bulgaria) & limonene (77.7-89.4\%) & {$[50]$} \\
\hline C. canadensis aerial parts EO (Łódź, Poland) & limonene $(76.3 \%)$ & [51] \\
\hline C. canadensis aerial parts EO (Alps, France) & limonene $(83.2 \%)$ & [51] \\
\hline
\end{tabular}


Table 2. Cont.

\begin{tabular}{|c|c|c|}
\hline Conyza Species (Collection Site) & Major Components (>5\%) & Ref. \\
\hline C. canadensis aerial parts EO (Rome, Italy) & limonene $(70.3 \%),(E)-\beta$-ocimene $(5.5 \%)$ & [51] \\
\hline C. canadensis aerial parts EO (Seville, Spain) & limonene $(51.4 \%),(E)$ - $\beta$-ocimene $(13.4 \%)$, trans- $\alpha$-bergamotene $(11.9 \%)$ & [51] \\
\hline C. canadensis aerial parts EO (Belgium) & $\begin{array}{l}\text { limonene }(68.0 \%),(E) \text { - } \beta \text {-ocimene }(5.1 \%) \text {, trans- } \alpha \text {-bergamotene }(5.4 \%) \text {, } \\
\text { germacrene } \mathrm{D}(7.3 \%)(\mathrm{Z}, \mathrm{Z}) \text {-matricaria ester }(6.1 \%)\end{array}$ & [51] \\
\hline C. canadensis aerial parts EO (Plovdiv, Bulgaria) & limonene $(87.9 \%)$ & [51] \\
\hline C. canadensis aerial parts EO (Vilnius, Lithuania) & limonene $(77.7 \%)$, trans- $\alpha$-bergamotene (5.5\%) & [51] \\
\hline C. canadensis aerial parts EO (Israel) & limonene (54.9\%), (Z)- $\beta$-farnesene (6.3\%) $(\mathrm{Z}, \mathrm{Z})$-matricaria ester $(7.7 \%)$ & [51] \\
\hline C. canadensis aerial parts EO (Kerman, Iran) & $\begin{array}{l}\text { myrcene }(8.9 \%) \text {, limonene }(12.3 \%),(E)-\beta \text {-farnesene }(14.6 \%) \text {, ar-curcumene }(7.8 \%) \text {, } \\
\text { zingiberene }(5.5 \%) \text {, spathulenol }(14.1 \%) \text {, isospathulenol }(7.7 \%) \text {, phytol }(7.3 \%)\end{array}$ & [52] \\
\hline C. canadensis aerial parts EO (Athens, Greece) & $\beta$-pinene $(9.5 \%)$, limonene $(57.3 \%)$, matricaria ester $(14.4 \%)$ & [43] \\
\hline C. canadensis aerial parts EO (Korea) & limonene $(68.3 \%),(E)$ - $\beta$-ocimene $(15.9 \%)^{b}$ & [53] \\
\hline C. canadensis EO (China) & $\begin{array}{l}\text { limonene }(14.8 \%) \text {, epi-bicyclosesquiphellandrene }(11.0 \%), \mathrm{C}_{7} \mathrm{H}_{30} \mathrm{~B}_{4} \mathrm{Si}(25.1 \%)^{\mathrm{c}} \text {, } \\
\text { 1-phenyl-1-nonyne }(7.3 \%)\end{array}$ & [54] \\
\hline C. canadensis aerial parts EO (Szeged, Hungary) & limonene $(79.2 \%)$ & [55] \\
\hline C. canadensis aerial parts EO (Manavgat, Antalya, Turkey) & $\beta$-pinene $(9.7 \%)$, limonene (28.1\%), spathulenol (16.3\%) & [56] \\
\hline C. canadensis aerial parts EO & $\beta$-pinene $(8.8 \%)$, limonene (41.5\%), (Z)-lachnophyllum ester (5.5\%) & this work \\
\hline C. sumatrensis aerial parts EO (Rondôndia state, Brazil) & $\begin{array}{l}\text { sabinene }(5.3 \%) \text {, limonene }(22.9 \%),(E) \text { - } \beta \text {-ocimene }(5.0 \%),(E) \text { - } \beta \text {-farnesene }(5.3 \%) \text {, } \\
\text { (Z)-lachnophyllum ester }(43.7 \%)\end{array}$ & [57] \\
\hline C. sumatrensis leaf EO (N'gorato village, Côte d'Ivoire) & $\begin{array}{l}\text { limonene }(13.0 \%),(E)-\beta \text {-ocimene }(6.5 \%),(E) \text {-caryophyllene }(10.5 \%) \text {, } \\
(E) \text { - } \beta \text {-farnesene }(17.0 \%),(Z) \text {-lachnophyllum ester }(5.9 \%) \text {, germacrene D }(13.6 \%) \text {, } \\
\text { bicyclogermacrene }(5.2 \%)\end{array}$ & [58] \\
\hline C. sumatrensis leaf EO (Monastir, Tunisia) & matricaria ester $(7.5 \%)$, spathulenol $(13.8 \%)$, caryophyllene oxide $(20.5 \%)$ & [59] \\
\hline C. sumatrensis aerial parts EO & $\begin{array}{l}\text { limonene }(25.5 \%),(E) \text {-caryophyllene }(5.5 \%),(E)-\beta \text {-farnesene }(6.7 \%) \text {, } \\
\text { (Z)-lachnophyllum ester }(20.7 \%) \text {, spathulenol }(5.2 \%) \text {, caryophyllene oxide }(5.8 \%)\end{array}$ & this work \\
\hline
\end{tabular}

${ }^{a}$ The identification of this compound is uncertain; it is not found in the Dictionary of Natural Products [60]. ${ }^{\mathrm{b}}$ This compound was listed as $\delta$-3-carene, but the retention time is more
consistent with $(E)-\beta$-ocimene rather than $\delta$-3-carene. ${ }^{c}$ The identification of this compound ( $2,3-\mu$-trimethylsilyl-C, $C^{\prime}$-dimethyl-4,5-dicarba-nido-hexaborane) is not correct; the compound

listed is not a natural product. 


\subsection{Mosquito Larvicidal Activity}

The mosquito larvicidal activities of the Conyza essential oils are summarized in Table 3. The essential oil of $C$. canadensis showed the best larvicidal activity against both Ae. aegypti $\left(24-\mathrm{h} \mathrm{LC}{ }_{50}=9.80 \mu \mathrm{g} / \mathrm{mL}\right)$ and Ae. albopictus $\left(24-\mathrm{h} \mathrm{LC}_{50}=18.0 \mu \mathrm{g} / \mathrm{mL}\right)$ and good larvicidal activity against $C x$. quinquefasciatus $\left(24-\mathrm{h} \mathrm{LC}_{50}=39.4 \mu \mathrm{g} / \mathrm{mL}\right)$. Conyza sumatrensis essential oil also showed good larvicidal activity against the three mosquito species $\left(24-\mathrm{h} \mathrm{LC}_{50}=21.7,19.1\right.$, and $26.7 \mu \mathrm{g} / \mathrm{mL}$, respectively, for Ae. aegypti, Ae. albopictus, and Cx. quinquefasciatus). Conyza bonariensis essential oil was less active $\left(24-\mathrm{h} \mathrm{LC}_{50}=69.7,81.1\right.$ and $130.0 \mu \mathrm{g} / \mathrm{mL}$ against Ae. aegypti, Ae. albopictus, and $C x$. quinquefasciatus, respectively).

The larvicidal activities of Conyza essential oils roughly coincides with the concentration of limonene in the samples $(41.5 \%, 25.5 \%$, and $0.2 \%$, respectively, for C. canadensis, C. sumatrensis, and C. bonariensis), and this relationship is borne out in a principle component analysis based on the major essential oil components (limonene, allo-aromadendrene, $(Z)$-lachnophyllum ester, caryophyllene oxide, $\beta$-caryophyllene, $\beta$-pinene, $(E)$ - $\beta$-farnesene, spathulenol, and $\alpha$-humulene, along with the 24 -h larvicidal activities) (Figure 1). Limonene has shown excellent larvicidal activities against Ae. aegypti (24-h $\left.\mathrm{LC}_{50}=17.7 \mu \mathrm{g} / \mathrm{mL}\right)$ and $C x$. quinquefasciatus $\left(24-\mathrm{h} \mathrm{LC}_{50}=31.6 \mu \mathrm{g} / \mathrm{mL}\right)$ (Table 3) as well as Ae. albopictus ( $\mathrm{LC}_{50}$ 10.8-41.8 $\mu \mathrm{g} / \mathrm{mL}$ ) [34]. Consistent with these results, Zeng and co-workers found the larvicidal activity of $C$. canadensis from China $(14.8 \%$ limonene) to be $56.9 \mu \mathrm{g} / \mathrm{mL}$ and $32.1 \mu \mathrm{g} / \mathrm{mL}$ against Ae. albopictus and Cx. quinquefasciatus, respectively [54]. These workers also appreciated the remarkable larvicidal activity and noted that $C$. canadensis essential oil has a potential for further development. Furthermore, Citrus peel oils, rich in limonene, have also shown remarkable larvicidal activities against Ae. albopictus [61] and Cx. quinquefasciatus [62].

Table 3. Mosquito larvicidal activity and insecticidal activity of Conyza essential oils.

\begin{tabular}{|c|c|c|c|c|c|}
\hline \multirow[b]{2}{*}{$\begin{array}{l}\text { Essential Oil or } \\
\text { Major Compound }\end{array}$} & \multicolumn{3}{|c|}{$24 \mathrm{~h}$} & \multirow[b]{2}{*}{$p$} & \multirow[b]{2}{*}{ Slope } \\
\hline & $\mathrm{LC}_{50}(95 \%$ Limits $), \mu \mathrm{g} / \mathrm{mL}$ & $\mathrm{LC}_{90}(95 \%$ Limits), $\mu \mathrm{g} / \mathrm{mL}$ & $x^{2}$ & & \\
\hline \multicolumn{6}{|c|}{ Aedes aegypti } \\
\hline C. bonariensis & $69.71(64.82-75.36)$ & $88.61(82.13-97.54)$ & 9.39 & 0.009 & 9.45 \\
\hline C. canadensis & $9.801(8.730-10.986)$ & $23.27(19.93-28.36)$ & 8.70 & 0.069 & 12.18 \\
\hline C. sumatrensis & $21.74(20.16-23.36)$ & $31.02(28.29-35.50)$ & 0.131 & 0.988 & 7.98 \\
\hline$\beta$-Pinene & $23.63(22.16-25.33)$ & $32.12(29.47-36.00)$ & 0.225 & 0.994 & 7.69 \\
\hline Limonene & $17.66(16.45-18.97)$ & $23.62(22.03-25.73)$ & 0.784 & 0.941 & 10.68 \\
\hline (E)-Caryophyllene & $70.80(65.49-76.69$ & $107.2(98.4-118.6)$ & 4.08 & 0.395 & 12.75 \\
\hline$\alpha$-Humulene & $53.05(48.69-58.08)$ & $82.78(75.81-91.87)$ & 15.9 & 0.003 & 12.79 \\
\hline Caryophyllene oxide & $136.6(129.2-143.9)$ & $180.2(171.4-191.2)$ & 30.1 & 0.000 & 12.37 \\
\hline Permethrin control & $0.000643(0.000551-0.00753)$ & $0.00246(0.00192-0.00344)$ & 12.5 & 0.006 & 11.57 \\
\hline \multicolumn{6}{|c|}{ Aedes albopictus a } \\
\hline C. bonariensis & $81.13(74.61-87.97)$ & $127.1(117.5-139.9)$ & 0.395 & 0.821 & 11.44 \\
\hline C. canadensis & $18.04(16.71-19.52)$ & $26.20(24.22-28.82)$ & 1.46 & 0.834 & 11.30 \\
\hline C. sumatrensis & $19.13(17.73-20.66)$ & $27.49(25.41-30.38)$ & 3.19 & 0.364 & 9.97 \\
\hline Permethrin control & $0.0024(0.0021-0.0026)$ & $0.0042(0.0038-0.0049)$ & 4.64 & 0.031 & 8.45 \\
\hline \multicolumn{6}{|c|}{ Culex quinquefasciatus } \\
\hline C. bonariensis & $130.0(122.5-138.8)$ & $178.4(165.6-197.2)$ & 0.675 & 0.713 & 8.97 \\
\hline C. canadensis & $39.37(36.83-42.00)$ & $52.29(49.04-56.56)$ & 0.493 & 0.974 & 10.49 \\
\hline C. sumatrensis & $26.74(24.80-29.20)$ & $36.83(33.56-41.92)$ & 8.97 & 0.030 & 7.96 \\
\hline$\beta$-Pinene & $30.46(28.21-33.21)$ & $41.58(38.10-46.58)$ & 0.399 & 0.983 & 9.38 \\
\hline Limonene & $31.63(29.37-34.50)$ & $41.51(38.03-46.78)$ & 0.874 & 0.928 & 8.23 \\
\hline (E)-Caryophyllene & $165.4(157.5-174.0)$ & $220.6(207.8-238.5)$ & 10.0 & 0.040 & 9.91 \\
\hline$\alpha$-Humulene & $108.3(101.4-115.5)$ & $158.2(148.5-170.5)$ & 1.0 & 0.910 & 13.32 \\
\hline Caryophyllene oxide & $98.52(90.70-108.68)$ & $144.5(129.6-165.7)$ & 1.60 & 0.809 & 9.20 \\
\hline Permethrin control & $0.0165(0.0149-0.0181)$ & $0.0305(0.0266-0.0367)$ & 5.24 & 0.073 & 10.12 \\
\hline \multicolumn{6}{|c|}{ Diplonychus rusticus a } \\
\hline C. canadensis & $135.7(129.3-142.8)$ & $182.5(172.6-195.5)$ & 7.78 & 0.051 & 12.35 \\
\hline C. sumatrensis & $111.0(106.1-116.7)$ & $137.0(129.5-147.6)$ & 16.1 & 0.001 & 9.85 \\
\hline
\end{tabular}


Table 3. Cont.

\begin{tabular}{|c|c|c|c|c|c|}
\hline \multirow[b]{2}{*}{$\begin{array}{l}\text { Essential Oil or } \\
\text { Major Compound }\end{array}$} & \multicolumn{3}{|c|}{$48 \mathrm{~h}$} & \multirow[b]{2}{*}{$p$} & \multirow[b]{2}{*}{ Slope } \\
\hline & $\mathrm{LC}_{50}(95 \%$ Limits $), \mu \mathrm{g} / \mathrm{mL}$ & $\mathrm{LC}_{90}(95 \%$ Limits),$\mu \mathrm{g} / \mathrm{mL}$ & $x^{2}$ & & \\
\hline \multicolumn{6}{|c|}{ Aedes aegypti } \\
\hline C. bonariensis & $63.85(59.07-70.75)$ & $81.84(74.16-94.79)$ & 3.43 & 0.180 & 6.89 \\
\hline C. canadensis & $7.091(6.099-8.141)$ & $22.46(18.63-28.59)$ & 5.98 & 0.201 & 11.63 \\
\hline C. sumatrensis & $22.52(21.18-23.87)$ & $29.00(27.23-31.68)$ & 0.0488 & 0.997 & 10.12 \\
\hline$\beta$-Pinene & $22.91(21.29-24.85)$ & $31.37(29.03-35.03)$ & 0.323 & 0.988 & 9.08 \\
\hline Limonene & $17.43(16.24-18.74)$ & $23.17(21.58-25.28)$ & 0.664 & 0.956 & 10.48 \\
\hline (E)-Caryophyllene & $65.92(60.45-72.08)$ & $106.4(98.4-116.7)$ & 14.2 & 0.007 & 13.10 \\
\hline$\alpha$-Humulene & $46.25(42.27-50.94)$ & $74.14(67.47-82.99)$ & 19.2 & 0.001 & 12.21 \\
\hline Caryophyllene oxide & $120.2(112.7-127.5)$ & $165.4(156.4-176.6)$ & 19.8 & 0.001 & 12.34 \\
\hline Permethrin control & $0.000575(0.000483-0.00688)$ & $0.00281(0.00208-0.00423)$ & 5.29 & 0.152 & 10.93 \\
\hline \multicolumn{6}{|c|}{ Aedes albopictus a } \\
\hline C. bonariensis & $69.42(63.20-75.93)$ & $113.2(103.8-125.8)$ & 3.10 & 0.212 & 10.72 \\
\hline C. canadensis & $15.12(13.93-16.47)$ & $22.67(20.84-25.09)$ & 7.23 & 0.124 & 12.22 \\
\hline C. sumatrensis & $18.43(17.05-19.93)$ & $26.76(24.71-29.58)$ & 4.25 & 0.236 & 8.44 \\
\hline \multicolumn{6}{|c|}{ Culex quinquefasciatus } \\
\hline C. bonariensis & $108.1(101.4-115.1)$ & $152.1(142.4-165.1)$ & 2.32 & 0.313 & 10.84 \\
\hline C. canadensis & $29.81(27.33-32.68)$ & $47.06(43.03-52.39)$ & 14.5 & 0.006 & 12.17 \\
\hline C. sumatrensis & $22.95(21.22-25.08)$ & $33.06(30.07-37.60)$ & 2.38 & 0.498 & 9.37 \\
\hline$\beta$-Pinene & $28.36(26.20-31.19)$ & $39.01(35.41-44.50)$ & 2.41 & 0.661 & 8.39 \\
\hline Limonene & $29.15(26.89-31.98)$ & $40.83(37.19-46.07)$ & 7.05 & 0.133 & 9.50 \\
\hline (E)-Caryophyllene & $138.5(129.3-148.5)$ & $215.3(200.1-234.9)$ & 13.5 & 0.009 & 13.11 \\
\hline$\alpha$-Humulene & $87.81(81.14-94.89)$ & $140.0(130.0-152.7)$ & 9.80 & 0.044 & 13.50 \\
\hline Caryophyllene oxide & $95.19(86.69-106.26)$ & $141.0(127.6-160.8)$ & 4.01 & 0.405 & 10.12 \\
\hline \multicolumn{6}{|c|}{ Diplonychus rusticus a } \\
\hline C. canadensis & $124.0(118.0-130.4)$ & $165.0(156.1-176.6)$ & 1.17 & 0.760 & 12.17 \\
\hline C. sumatrensis & $107.8(103.1-113.4)$ & $133.6(126.1-144.4)$ & 8.07 & 0.045 & 9.37 \\
\hline
\end{tabular}

${ }^{a}$ Aedes albopictus and Diplonychus rusticus were obtained from the wild; the limited numbers of organisms available precluded screening of individual components on these two insect species.

Other components in the Conyza essential oils likely contribute to the mosquito larvicidal effects. Conyza bonariensis was rich in (E)-caryophyllene (13.3\%) and caryophyllene oxide $(12.2 \%)$, but both of these compounds have been found to have weak larvicidal activities against Ae. aegypti (24-h $\mathrm{LC}_{50}=70.8$ and $137 \mu \mathrm{g} / \mathrm{mL}$, respectively (Table 3). On the other hand, $\beta$-pinene, a major component of $C$. canadensis essential oil $(8.8 \%)$, has shown larvicidal activity against Ae. aegypti (24-h LC $50=23.6 \mu \mathrm{g} / \mathrm{mL}), C x$. quinquefasciatus (24-h LC $50=30.5 \mu \mathrm{g} / \mathrm{mL})$ (Table 3), and Ae. albopictus [61]. In addition, synergy between essential oil components may also be important $[63,64]$. Scalerandi and coworkers have found that the housefly (Musca domestica) metabolizes the major components in an essential oil, but leaves the minor components to act as toxicants [65].

In order to assess the potential detrimental impact of the Conyza essential oils on beneficial aquatic species, the insecticidal activity was assessed against the water bug, Diplonychus rusticus, an insect predator of mosquito larvae [66]. Both C. canadensis and C. sumatrensis essential oils were substantially less toxic to $D$. rusticus than they were to the mosquito larvae. 


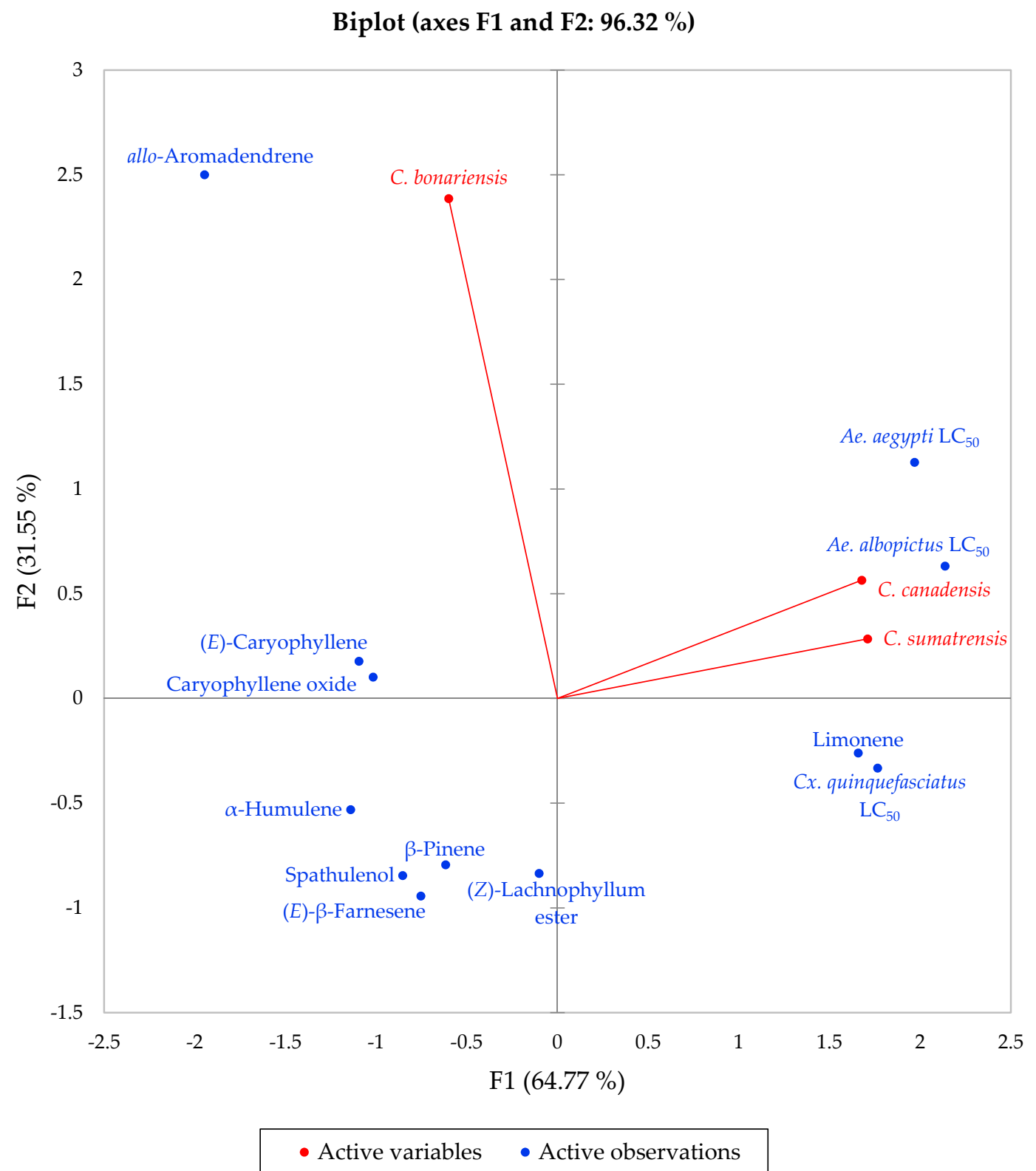

Figure 1. Principal component biplot of PC1 and PC2 scores and loadings demonstrating the relationships between Conyza essential oil major components and larvicidal activities.

\section{Materials and Methods}

\subsection{Chemicals}

Chemicals used for this study, dimethylsulfoxide (DMSO), $\beta$-pinene, limonene, (E)-caryophyllene, $\alpha$-humulene, caryophyllene oxide, dichloromethane, and permethrin, were obtained from Sigma-Aldrich (St. Louis, MO, USA) and used as received without further purification.

\subsection{Plant Material}

The three Conyza species were collected from Bach Ma National Park, Thue Thien Hue province $\left(16^{\circ} 11^{\prime} 34^{\prime \prime} \mathrm{N}, 107^{\circ} 51^{\prime} 12^{\prime \prime} \mathrm{E}\right)$ in April 2020. The plants were identified by Dr. Do Ngoc Dai and Dr. Le Thi Huong. Voucher specimens, LTH129 (Conyza canadensis), LTH130 (Conyza sumatrensis), and LTH131 (Conyza bonariensis) have been deposited in the Pedagogical Institute of Science, Vinh University. Four-kg 
samples of fresh aerial parts (leaves, stems, and flowers) of each of the plants were shredded and hydrodistilled for $4 \mathrm{~h}$ using a Clevenger-type apparatus.

\subsection{Gas Chromatography-Mass Spectrometry}

The Conyza essential oils were analyzed by GC-MS as previously described [67]: Shimadzu GCMS-QP2010 Ultra, electron impact (EI) mode, electron energy $=70 \mathrm{eV}$, scan range $=40-400$ atomic mass units, scan rate $=3.0$ scans/s, ZB-5 fused silica capillary column $(30 \mathrm{~m} \times 0.25 \mathrm{~mm}, 0.25 \mu \mathrm{m}$ film thickness), He carrier gas, $552 \mathrm{kPa}$ column head pressure, and $1.37 \mathrm{~mL} / \mathrm{min}$ flow rate. Injector temperature was $250^{\circ} \mathrm{C}$ and the ion source temperature was $200^{\circ} \mathrm{C}$. The GC oven temperature program was programmed for $50^{\circ} \mathrm{C}$ initial temperature, temperature increased at a rate of $2{ }^{\circ} \mathrm{C} / \mathrm{min}$ to $260^{\circ} \mathrm{C}$. A $5 \%$ w/v solution of the sample in $\mathrm{CH}_{2} \mathrm{Cl}_{2}$ was prepared and $0.1 \mu \mathrm{L}$ was injected with a splitting mode (30:1). Identification of the oil components was based on their retention indices determined by reference to a homologous series of $n$-alkanes, and by comparison of their mass spectral fragmentation patterns with those reported in the databases [36-39].

\subsection{Mosquito Larvicidal Assay}

Mosquito larvicidal activity was carried out on Ae. aegypti, Ae. albopictus, and Cx. quinquefasciatus as previously described [67]: For the assay, $1 \%$ stock solutions of each essential oil in dimethylsulfoxide (DMSO) were prepared, and aliquots of the stock solutions were placed in 500-mL beakers and added to water that contained 20 larvae (fourth instar). With each experiment, a set of controls using DMSO was also run for comparison. Mortality was recorded after $24 \mathrm{~h}$ and again after $48 \mathrm{~h}$ of exposure during which no nutritional supplement was added. The experiments were carried out $25 \pm 2{ }^{\circ} \mathrm{C}$. Each test was conducted with four replicates with three concentrations $(50,25$, and $12.5, \mu \mathrm{g} / \mathrm{mL}$ for $C$. canadensis and C. sumatrensis; 150,100 , and $50 \mu \mathrm{g} / \mathrm{mL}$ for C. bonariensis). Permethrin was used as a positive control.

\subsection{Non-Target Insecticidal Assay}

The Diplonychus rusticus adults were collected in the field and maintained in glass tanks ( $60 \mathrm{~cm}$ long $\times 50 \mathrm{~cm}$ wide) containing water at $25{ }^{\circ} \mathrm{C}$ with a water depth of $20 \mathrm{~cm}$. The essential oils were tested at concentrations of 200, 150, 100, 75, 50, and $25 \mu \mathrm{g} / \mathrm{mL}$. Four replicates were performed for each concentration. Twenty D. rusticus adults were introduced into each solution. The non-target organism was observed for mortality after $24 \mathrm{~h}$ and $48 \mathrm{~h}$ exposure.

\subsection{Data Analysis}

The mortalities were recorded $24 \mathrm{~h}$ and $48 \mathrm{~h}$ after treatment. The data obtained were subjected to log-probit analysis [68] to obtain $\mathrm{LC}_{50}$ values, $\mathrm{LC}_{90}$ values, $95 \%$ confidence limits, and chi square values using Minitab ${ }^{\circledR} 18$ (Minitab Inc., State College, PA, USA). For the principal component analysis (PCA), the 9 major components (limonene, allo-aromadendrene, (Z)-lachnophyllum ester, caryophyllene oxide, (E)-caryophyllene, $\beta$-pinene, (E)- $\beta$-farnesene, spathulenol, and $\alpha$-humulene), and the 24 -h larvicidal activities against $A$ e. aegypti, Ae. albopictus, and $C x$. quinquefasciatus were taken as variables using a Pearson correlation matrix using XLSTAT Premium, version 2018.5 (Addinsoft, Paris, France). A total of 33 data (11 variables $\times 3$ samples) were used for the PCA.

\section{Conclusions}

Invasive plant species are generally considered to be ecologically and detrimental with potential economic impacts, and the control or eradication of invasive plant species can be prohibitively costly. However, identification of beneficial uses of invasive plants could be economically advantageous and aid in the control of the species. Conyza spp., as well as Erechtites spp. [34], Crassocephalum crepidioides [35], and Severinia monophylla [33], are invasive weeds in Vietnam, and essential oils from these plants have demonstrated promising mosquito larvicidal activities. The plant materials are readily available and 
harvesting of these weeds may provide economically valuable "cash crops" as well as serve as a means for ecological remediation. Note that C. bonariensis [69], C. canadensis [70], and C. sumatrensis [71] have all shown resistance to the commonly used herbicide glyphosate, so herbicidal control of these weeds is impractical as well as environmentally detrimental. Further research on potential formulations (e.g., nanoemulsions or essential oil-loaded nanoparticles) [72] for field use of these promising essential oils is warranted.

Author Contributions: Conceptualization, N.H.H.; methodology, T.M.H., P.S., and W.N.S.; software, P.S.; validation, T.M.H., D.N.D., P.S., and W.N.S.; formal analysis, P.S. and W.N.S.; investigation, T.M.H., D.N.D., H.V.C., D.V.H., T.A.T., L.T.H., N.H.H., and V.T.H.; resources, T.M.H.; data curation, W.N.S.; writing-original draft preparation, W.N.S.; writing-review and editing, T.M.H., D.N.D., N.H.H., P.S., and W.N.S.; supervision, N.H.H.; project administration, T.M.H.; funding acquisition, T.M.H. All authors have read and agreed to the published version of the manuscript.

Funding: Please add: This research is funded by Vietnam National Foundation for Science and Technology Development (NAFOSTED) under grant number: 106.03-2019.315.

Acknowledgments: P.S. and W.N.S. participated in this work as part of the activities of the Aromatic Plant Research Center (APRC, https://aromaticplant.org/).

Conflicts of Interest: The authors declare no conflict of interest.

\section{References}

1. Kim Lien, P.T.; Briant, L.; Tang, T.B.; Trang, B.M.; Gavotte, L.; Cornillot, E.; Duoc, V.T.; Duong, T.N.; Frutos, R.; Nga, P.T. Surveillance of dengue and chikungunya infection in Dong Thap, Vietnam: A 13-month study. Asian Pac. J. Trop. Med. 2016, 9, 39-43. [CrossRef] [PubMed]

2. Pham Thi, K.L.; Briant, L.; Gavotte, L.; Labbe, P.; Perriat-Sanguinet, M.; Cornillot, E.; Vu, T.D.; Nguyen, T.Y.; Tran, V.P.; Nguyen, V.S.; et al. Incidence of dengue and chikungunya viruses in mosquitoes and human patients in border provinces of Vietnam. Parasit. Vectors 2017, 10, 556. [CrossRef] [PubMed]

3. Powell, J.R. Mosquito-borne human viral diseases: Why Aedes aegypti? Am. J. Trop. Med. Hyg. 2018, 98, 1563-1565. [CrossRef] [PubMed]

4. Lourenço de Oliveira, R.; Vazeille, M.; de Filippis, A.M.B.; Failloux, A.B. Large genetic differentiation and low variation in vector competence for dengue and yellow fever viruses of Aedes albopictus from Brazil, the United States, and the Cayman Islands. Am. J. Trop. Med. Hyg. 2003, 69, 105-114. [CrossRef] [PubMed]

5. Lambrechts, L.; Scott, T.W.; Gubler, D.J. Consequences of the expanding global distribution of Aedes albopictus for dengue virus transmission. PLoS Negl. Trop. Dis. 2010, 4, e646. [CrossRef]

6. Vazeille, M.; Moutailler, S.; Coudrier, D.; Rousseaux, C.; Khun, H.; Huerre, M.; Thiria, J.; Dehecq, J.S.; Fontenille, D.; Schuffenecker, I.; et al. Two Chikungunya isolates from the outbreak of La Reunion (Indian Ocean) exhibit different patterns of infection in the mosquito, Aedes albopictus. PLoS ONE 2007, 2, e1168. [CrossRef]

7. Wong, P.-S.J.; Li, M.I.; Chong, C.-S.; Ng, L.-C.; Tan, C.-H. Aedes (Stegomyia) albopictus (Skuse): A potential vector of Zika virus in Singapore. PLoS Negl. Trop. Dis. 2013, 7, e2348. [CrossRef]

8. Albuquerque, C.M.R.; Cavalcanti, V.M.S.; Melo, M.A.V.; Verçosa, P.; Regis, L.N.; Hurd, H. Bloodmeal microfilariae density and the uptake and establishment of Wuchereria bancrofti infections in Culex quinquefasciatus and Aedes aegypti. Mem. Inst. Oswaldo Cruz 1999, 94, 591-596. [CrossRef]

9. Turell, M.J. Members of the Culex pipiens complex as vectors of viruses. J. Am. Mosq. Control Assoc. 2012, 28, 123-127. [CrossRef]

10. van den Hurk, A.F.; Hall-Mendelin, S.; Jansen, C.C.; Higgs, S. Zika virus and Culex quinquefasciatus mosquitoes: A tenuous link. Lancet Infect. Dis. 2017, 17, 1014-1016. [CrossRef]

11. Thebaud, C.; Abbott, R.J. Characterization of invasive Conyza species (Asteraceae) in Europe: Quantitative trait and isozyme analysis. Am. J. Bot. 1995, 82, 360-368. [CrossRef]

12. Prieur-Richard, A.-H.; Lavorel, S.; Grigulis, K.; Dos Santos, A. Plant community diversity and invasibility by exotics: Invasion of Mediterranean old fields by Conyza bonariensis and Conyza canadensis. Ecol. Lett. 2000, 3, 412-422. [CrossRef] 
13. Bajwa, A.A.; Sadia, S.; Ali, H.H.; Jabran, K.; Peerzada, A.M.; Chauhan, B.S. Biology and management of two important Conyza weeds: A global review. Environ. Sci. Pollut. Res. 2016, 23, 24694-24710. [CrossRef] [PubMed]

14. Wu, H.; Walker, S.; Rollin, M.J.; Tan, D.K.Y.; Robinson, G.; Werth, J. Germination, persistence, and emergence of flaxleaf fleabane (Conyza bonariensis [L.] Cronquist). Weed Biol. Manag. 2007, 7, 192-199. [CrossRef]

15. Wu, H. The biology of Australian weeds. Plant Prot. Quart. 2007, 22, 122-131.

16. Pruski, J.F.; Sancho, G. Conyza sumatrensis var. leiotheca (Compositae: Astereae), a new combination for a common Neotropical weed. Novon 2006, 16, 96-101. [CrossRef]

17. Raghubanshi, A.S.; Rai, L.C.; Gaur, J.P.; Singh, J.S. Invasive alien species and biodiversity in India. Curr. Sci. 2005, 88, 539-540.

18. Weidenhamer, J.D.; Callaway, R.M. Direct and indirect effects of invasive plants on soil chemistry and ecosystem function. J. Chem. Ecol. 2010, 36, 59-69. [CrossRef]

19. Pimentel, D.; Zuniga, R.; Morrison, D. Update on the environmental and economic costs associated with alien-invasive species in the United States. Ecol. Econ. 2005, 52, 273-288. [CrossRef]

20. Kettenring, K.M.; Adams, C.R. Lessons learned from invasive plant control experiments: A systematic review and meta-analysis. J. Appl. Ecol. 2011, 48, 970-979. [CrossRef]

21. Pasko, S.; Goldberg, J. Review of harvest incentives to control invasive species. Manag. Biol. Invasions 2014, 5, 263-277. [CrossRef]

22. Stocker, R.K. Mechanical harvesting of Melaleuca quinquenervia in Lake Okeechobee, Florida. Ecol. Eng. 1999, 12, 373-386. [CrossRef]

23. Carson, B.D.; Lishawa, S.C.; Tuchman, N.C.; Monks, A.M.; Lawrence, B.A.; Albert, D.A. Harvesting invasive plants to reduce nutrient loads and produce bioenergy: An assessment of Great Lakes coastal wetlands. Ecosphere 2018, 9, e02320. [CrossRef]

24. Benelli, G.; Pavela, R.; Cianfaglione, K.; Nagy, D.U.; Canale, A.; Maggi, F. Evaluation of two invasive plant invaders in Europe (Solidago canadensis and Solidago gigantea) as possible sources of botanical insecticides. J. Pest Sci. 2019, 92, 805-821. [CrossRef]

25. Kamrin, M.A. Pesticide Profiles: Toxicity, Environmental Impact, and Fate; CRC Press: Boca Raton, FL, USA, 1997; ISBN 0-56670-190-2.

26. Goulson, D. An overview of the environmental risks posed by neonicotinoid insecticides. J. Appl. Ecol. 2013, 50, 977-987. [CrossRef]

27. Cuervo-Para, J.A.; Romero Cortés, T.; Ramirez-Lepe, M. Mosquito-borne Diseases, Pesticides Used for Mosquito Control, and Development of Resistance to Insecticides. In Insecticides Resistance; Trdan, S., Ed.; IntechOpen: London, UK, 2016; pp. 111-134. ISBN 978-953-51-2258-6.

28. Silva, W.J.; Dória, G.A.A.; Maia, R.T.; Nunes, R.S.; Carvalho, G.A.; Blank, A.F.; Alves, P.B.; Marçal, R.M.; Cavalcanti, S.C.H. Effects of essential oils on Aedes aegypti larvae: Alternatives to environmentally safe insecticides. Bioresource Technol. 2008, 99, 3251-3255. [CrossRef]

29. Benelli, G. Research in mosquito control: Current challenges for a brighter future. Parasitol. Res. 2015, 114, 2801-2805. [CrossRef]

30. Masetti, A. The potential use of essential oils against mosquito larvae: A short review. Bull. Insectol. 2016, 69, 307-310.

31. Pavela, R.; Benelli, G. Essential oils as ecofriendly biopesticides? Challenges and constraints. Trends Plant Sci. 2016, 21, 1000-1007. [CrossRef]

32. Ntalli, N.; Koliopoulos, G.; Giatropoulos, A.; Menkissoglu-Spiroudi, U. Plant secondary metabolites against arthropods of medical importance. Phytochem. Rev. 2019, 18, 1255-1275. [CrossRef]

33. Satyal, P.; Hieu, H.V.; Chuong, N.T.H.; Hung, N.H.; Sinh, L.H.; Van The, P.; Tai, T.A.; Hien, V.T.; Setzer, W.N. Chemical composition, Aedes mosquito larvicidal activity, and repellent activity against Triatoma rubrofasciata of Severinia monophylla leaf essential oil. Parasitol. Res. 2019, 118, 733-742. [CrossRef]

34. Hung, N.H.; Satyal, P.; Hieu, H.V.; Chuong, N.T.H.; Dai, D.N.; Huong, L.T.; Tai, T.A.; Setzer, W.N. Mosquito larvicidal activity of the essential oils of Erechtites species growing wild in Vietnam. Insects 2019, 10, 47. [CrossRef] [PubMed] 
35. Hung, N.H.; Satyal, P.; Do, N.D.; Tai, T.A.; Huong, L.T.; Chuong, N.T.H.; Hieu, H.V.; Tuan, P.A.; Vuong, P.; Van Setzer, W.N. Chemical compositions of Crassocephalum crepidioides essential oils and larvicidal activities against Aedes aegypti, Aedes albopictus, and Culex quinquefasciatus. Nat. Prod. Commun. 2019, 14. [CrossRef]

36. Adams, R.P. Identification of Essential Oil Components by Gas Chromatography/Mass Spectrometry, 4th ed.; Allured Publishing: Carol Stream, IL, USA, 2007; ISBN 978-1-932633-21-4.

37. Mondello, L. FFNSC 3; Shimadzu Scientific Instruments: Columbia, MD, USA, 2016.

38. NIST17; National Institute of Standards and Technology: Gaithersburg, MD, USA, 2017.

39. Satyal, P. Development of GC-MS Database of Essential Oil Components by the Analysis of Natural Essential Oils and Synthetic Compounds and Discovery of Biologically Active Novel Chemotypes in Essential Oils. Ph.D. Thesis, University of Alabama in Huntsville, Huntsville, AL, USA, 2015.

40. Maia, J.G.S.; da Silva, M.H.L.; das Gracas, M.B.Z.; Andrade, E. Composition of the essential oils of Conyza bonariensis (L.) Cronquist. J. Essent. Oil Res. 2002, 14, 325-326. [CrossRef]

41. Souza, M.C.; Siani, A.C.; Ramos, M.F.S.; Menezes-de-lima, O., Jr.; Henriques, M.G.M.O. Evaluation of anti-inflammatory activity of essential oils from two Asteraceae species. Pharmazie 2003, 58, 582-586. [PubMed]

42. Barbosa, L.C.A.; Paula, V.F.; Azevedo, A.S.; Silva, E.A.M.; Nascimento, E.A. Essential oil composition from some plant parts of Conyza bonariensis (L.) Cronquist. Flavour Fragr. J. 2005, 20, 39-41. [CrossRef]

43. Tzakou, O.; Vagias, C.; Gani, A.; Yannitsaros, A. Volatile constituents of essential oils isolated at different growth stages from three Conyza species growing in Greece. Flavour Fragr. J. 2005, 20, 425-428. [CrossRef]

44. Urdampilleta, J.D.; Amat, A.G.; Bidau, C.J.; Koslobsky, N.K. Biosystematic and chemosystematic studies in five South American species of Conyza (Asteraceae). Bol. Soc. Argent. Bot. 2005, 40, 101-107.

45. Mabrouk, S.; Elaissi, A.; Ben Jannet, H.; Harzallah-Skhiri, F. Chemical composition of essential oils from leaves, stems, flower heads and roots of Conyza bonariensis L. from Tunisia. Nat. Prod. Res. 2011, 25, 77-84. [CrossRef]

46. Benzarti, A.; Hammami, S.; Piras, A.; Falconieri, D.; El Mokni, R.; M’Henni, M.F.; Marongiu, B.; Mighri, Z. Effects of different ecological conditions and extraction techniques on the quality of volatile oils from flaxleaf fleabane (Erigeron bonariensis L.). J. Med. Plant Res. 2013, 7, 3059-3065.

47. Araujo, L.; Moujir, L.M.; Rojas, J.; Carmona, J.; Rondón, M. Chemical composition and biological activity of Conyza bonariensis essential oil collected in Mérida, Venezuela. Nat. Prod. Commun. 2013, 8, 1175-1178. [CrossRef] [PubMed]

48. Musembei, R.; Joyce, K.J. Chemical composition and antibacterial activity of essential oil from Kenyan Conyza bonariensis (L.) Cronquist. Sci. Lett. 2017, 5, 180-185.

49. do Amaral, W.; Deschamps, C.; Biasi, L.A.; Bizzo, H.R.; Machado, M.P.; da Silva, L.E. Yield and chemical composition of the essential oil of species of the Asteraceae family from Atlantic Forest, South of Brazil. J. Essent. Oil Res. 2018, 30, 278-284. [CrossRef]

50. Stoyanova, A.; Georgiev, E.; Kermedchieva, D.; Lis, A.; Gora, J. Changes in the essential oil of Conyza canadensis (L.) Cronquist. during its vegetation. J. Essent. Oil Res. 2003, 15, 44-45. [CrossRef]

51. Lis, A.; Piggott, J.R.; Góra, J. Chemical composition variability of the essential oil of Conyza canadensis Cronq. Flavour Fragr. J. 2003, 18, 364-367. [CrossRef]

52. Rustaiyan, A.; Azar, P.A.; Moradalizadeh, M.; Masoudi, S.; Ameri, N. Volatile constituents of three Compositae herbs: Anthemis altissima L. var. altissima, Conyza canadensis (L.) Cronq. and Grantina aucheri Boiss. growing wild in Iran. J. Essent. Oil Res. 2004, 16, 579-581. [CrossRef]

53. Choi, H.-J.; Wang, H.-Y.; Kim, Y.-N.; Heo, S.-J.; Kim, N.-K.; Jeong, M.-S.; Park, Y.-H.; Kim, S. Composition and cytotoxicity of essential oil extracted by steam distillation from horseweed (Erigeron canadensis L.) in Korea. J. Korean Soc. Appl. Biol. Chem. 2008, 51, 55-59.

54. Zeng, D.-Q.; Peng, Y.-H.; Chen, F.-F.; Zhang, Y.; Liu, M. Insecticidal activity of essential oil derived from horseweed Conyza canadensis (L.) Cronq.against two mosquitoes and its volatile components. Acta Entomol. Sin. 2014, 57, 204-211.

55. Veres, K.; Csupor-Löffler, B.; Lázár, A.; Hohmann, J. Antifungal activity and composition of essential oils of Conyza canadensis herbs and roots. Sci. World J. 2012, 2012, 489646. [CrossRef]

56. Ayaz, F.; Küçükboyac1, N.; Demirci, B. Chemical composition and antimicrobial activity of the essential oil of Conyza canadensis (L.) Cronquist from Turkey. J. Essent. Oil Res. 2017, 29, 336-343. [CrossRef] 
57. Machado, S.M.F.; Militão, J.S.L.T.; Facundo, V.A.; Ribeiro, A.; de Morais, S.M.; de Alencar, J.W.; Braz Filho, R. Essential oil of Conyza sumatrensis (Retz) Walk. J. Essent. Oil Res. 1995, 7, 83-84. [CrossRef]

58. Boti, J.B.; Koukoua, G.; N'Guessan, T.Y.; Casanova, J. Chemical variability of Conyza sumatrensis and Microglossa pyrifolia from Côte d'Ivoire. Flavour Fragr. J. 2007, 22, 27-31. [CrossRef]

59. Mabrouk, S.; Salah, K.B.H.; Elaissi, A.; Jlaiel, L.; Ben Jannet, H.; Aouni, M.; Harzallah-Skhiri, F. Chemical composition and antimicrobial and allelopathic activity of Tunisian Conyza sumatrensis (Retz.) E. Walker essential oils. Chem. Biodivers. 2013, 10, 209-223. [CrossRef] [PubMed]

60. Dictionary of Natural Products on DVD; CRC Press: Boca Raton, FL, USA, 2019; ISBN 0-412-49150-8.

61. Giatropoulos, A.; Papachristos, D.P.; Kimbaris, A.; Koliopoulos, G.; Polissiou, M.G.; Emmanouel, N.; Michaelakis, A. Evaluation of bioefficacy of three Citrus essential oils against the dengue vector Aedes albopictus (Diptera: Culicidae) in correlation to their components enantiomeric distribution. Parasitol. Res. 2012, 111, 2253-2263. [CrossRef]

62. Manimaran, A.; Cruz, M.M.J.J.; Muthu, C.; Vincent, S.; Ignacimuthu, S. Larvicidal and knockdown effects of some essential oils against Culex quinquefasciatus Say, Aedes aegypti (L.) and Anopheles stephensi (Liston). Adv. Biosci. Biotechnol. 2012, 3, 855-862. [CrossRef]

63. Pavela, R. Acute toxicity and synergistic and antagonistic effects of the aromatic compounds of some essential oils against Culex quinquefasciatus Say larvae. Parasitol. Res. 2015, 114, 3835-3853. [CrossRef]

64. Tak, J.H.; Isman, M.B. Penetration-enhancement underlies synergy of plant essential oil terpenoids as insecticides in the cabbage looper, Trichoplusia ni. Sci. Rep. 2017, 7, 42432. [CrossRef]

65. Scalerandi, E.; Flores, G.A.; Palacio, M.; Defagó, M.T.; Carpinella, M.C.; Valladares, G.; Bertoni, A.; Palacios, S.M. Understanding synergistic toxicity of terpenes as insecticides: Contribution of metabolic detoxification in Musca domestica. Front. Plant Sci. 2018, 9, 1579. [CrossRef]

66. Saha, N.; Aditya, G.; Bal, A.; Saha, G.K. A comparative study of predation of three aquatic heteropteran bugs on Culex quinquefasciatus larvae. Limnology 2007, 8, 73-80. [CrossRef]

67. Huong, L.T.; Hung, N.H.; Dai, D.N.; Tai, T.A.; Hien, V.T.; Satyal, P.; Setzer, W.N. Chemical compositions and mosquito larvicidal activities of essential oils from Piper species. Molecules 2019, 24, 3871. [CrossRef]

68. Finney, D. Probit Analysis; Reissue, Ed.; Cambridge University Press: Cambridge, UK, 2009; ISBN 978-0521135900.

69. Travlos, I.S.; Chachalis, D. Glyphosate-resistant hairy fleabane (Conyza bonariensis) is reported in Greece. Weed Technol. 2010, 24, 569-573. [CrossRef]

70. Koger, C.H.; Poston, D.H.; Hayes, R.M.; Montgomery, R.F. Glyphosate-resistant horseweed (Conyza canadensis) in Mississippi. Weed Technol. 2004, 18, 820-825. [CrossRef]

71. Santos, G.; Oliveira, R.S., Jr.; Constantin, J.; Francischini, A.C.; Machado, M.F.P.S.; Mangolin, C.A.; Nakajima, J.N. Conyza sumatrensis: A new weed species resistant to glyphosate in the Americas. Weed Biol. Manag. 2014, 14, 106-114. [CrossRef]

72. Pavela, R.; Maggi, F.; Iannarelli, R.; Benelli, G. Plant extracts for developing mosquito larvicides: From laboratory to the field, with insights on the modes of action. Acta Trop. 2019, 193, 236-271. [CrossRef] [PubMed]

Sample Availability: Samples of the Conyza essential oils are no longer available.

(C) 2020 by the authors. Licensee MDPI, Basel, Switzerland. This article is an open access article distributed under the terms and conditions of the Creative Commons Attribution (CC BY) license (http://creativecommons.org/licenses/by/4.0/). 